\title{
AVALIAÇÃO EPIDEMIOLÓGICA COMPARATIVA DE HOSPITALIZAÇÕES POR SÍNDROME RESPIRATÓRIA AGUDA GRAVE (SRAG) E POR COVID-
}

19

\author{
COMPARATIVE EPIDEMIOLOGICAL EVALUATION OF HOSPITALIZATIONS \\ BY SEVERE ACUTE RESPIRATORY SYNDROME (SARS) AND COVID-19
}

Anna Maria Andrade Barbosa*, Vitória Lorrane Dos Santos, Bárbara De Oliveira Arantes, João Paulo Pires Caixêta, Antonio Márcio Teodoro Cordeiro Silva².

Pontifícia Universidade Católica de Goiás, Goiânia, GO, Brasil.

*annamandrade@icloud.com

\section{RESUMO}

A COVID-19 é uma doença respiratória, causada pelo SARS-CoV-2, que pode produzir desde um quadro assintomático até progredir para um estado grave. A SRAG ocorre devido à progressão de quadros de infecções respiratórias por vírus e vem ganhando destaque pelo aumento no Brasil causado pela COVID19. À luz desse panorama tem-se por objetivo avaliar se a pandemia de SARSCoV-2, no Brasil, resultou em alterações no padrão de hospitalizações de SRAG, comparando a evolução da COVID-19 e de SRAG, até a 35a semana epidemiológicas de 2020, e a frequência de hospitalização por SRAG nos dois anos anteriores por meio de um estudo epidemiológico, comparativo, das hospitalizações por SRAG, no período de 2018 a 2020, no Brasil, com informações referentes à tabulação e interpretação dos dados extraídos em 09 de setembro de 2020, do Monitoramento de Casos Reportados de Síndrome Respiratória Aguda Grave Hospitalizados, disponibilizado pela Fiocruz, em parceria com o Ministério da Saúde e a FGV. A análise demonstrou que a COVID-19 obteve o primeiro registro no Brasil na 9a semana epidemiológica com aumento exponencial de casos, e atingindo o maior registro de hospitalização simultâneas, por SRAG, na $19^{\text {a }}$ semana $(n=21.568$, no total e $n=8.545$, não contabilizando os casos confirmados de COVID-19). Esse valor foi 11,6 vezes superior ao observado do pico dos dois anos anteriores (2018, com $n=1.855$, e 2019, com $n=1.861$ ), e sem contabilizar os casos de COVID-19, foi 4,6 maior. A frequência de hospitalizações, por SRAG não COVID-19, estratificada por faixa etária, revelou que indivíduos de 0 a 5 anos de idade apresentaram a maior frequência de casos notificados no período analisado (média de $48,8 \%$, nos anos de 2018 e 2019), exceto em 2020, sendo esta entre maiores de 60 anos (46,8\%). Considerando somente casos de COVID-19, este índice subiu para $49,5 \%$. Destacam-se as diferenças entre os óbitos, anteriormente à pandemia, e, posteriormente, devido à SRAG, com aumento de 10 vezes na incidência de mortes não notificadas, como COVID-19, e 25 vezes nas confirmadas. Ademais, nota-se que a taxa de letalidade também aumentou, no período, sendo 2018 de 10,6\%; 2019 de 10,1\%; e 2020 de 24,9\%. Contabilizando apenas os notificados, como SRAG, esse valor caiu para $15,9 \%$ e, apenas os positivos para COVID-19, elevou-se para $33,1 \%$. Vale ressaltar, também, o aumento percentual das mortes na faixa etária maior de 60 anos, representando 43,9\%; 43,0\% e 73,3\%, 
respectivamente, nos anos avaliados. Portanto, o aumento da hospitalização, por SRAG, deve ser visto com olhar crítico, podendo, a causa ser decorrente da alta subnotificação de casos adjunto ao aumento de notificações de influenza devido à situação atual da pandemia, em que o paciente procura atendimento hospitalar pelo quadro similar de COVID-19 e influenza. Necessitam-se estudos mais aprofundados sobre esse aumento para avaliação mais criteriosa da situação de contágio e letalidade da doença, no país.

Palavras-chave: Brasil. COVID-19. Epidemiologia. Pandemia. SRGA. 